\title{
GRAMMATICA CONTRASTIVA, LESSICOGRAFIA E INSEGNAMENTO: VERBI TRANSITIVI / INTRANSITIVI E COMPLEMENTI
}

\author{
Adriana Padoan / Rossend Arqués Corominas \\ Universitat Autònoma de Barcelona
}

\begin{abstract}
Resumen: Como autores del Grande Dizionario di Spagnolo Zanichelli (2012) (GDS), nos hemos enfrentado a varias cuestiones referentes a la taxonomía gramatical del español y el italiano. En este artículo exponemos el modo en que hemos resuelto en la práctica lexicográfica dos problemas relevantes: el tratamiento de los complementos y los verbos transitivos / intransitivos. Proponemos asimismo unas breves reflexiones sobre la descripción gramatical en la enseñanza de la lengua como L2.
\end{abstract}

Palabras clave: lexicografía bilingüe, verbos transitivos / intransitivos, complementos, gramática contrastiva, enseñanza italiano / español L2

Abstract: As authors of the 'GrandeDizionario di Spagnolo Zanichelli' (2012) (GDS), we raised several questions on the description of grammatical voices in Spanish and Italian. In this article we present how we have dealt with lexicographic practice in relation to two issues: the treatment of objects and of transitive / intransitive verbs. Here we present some brief reflections on how we use grammatical descriptions in the teaching of Italian as a second language. Keywords: bilingual lexicography, transitive / intransitive verbs, complements, contrastive grammar, Italian / Spanish learning L2

\section{PREMESSA}

Nel nostro pluriennale lavoro lessicografico per la realizzazione del GDS 1 abbiamo incontrato difficoltà di varia natura (etichette dei limiti d'uso, termini

${ }^{1}$ Sigle e abbrevizioni usate in questo articolo: GDS = R. Arqués e A. Padoan, Grande Dizionario di Spagnolo, Bologna, Zanichelli, 2012; GRADIT = Grande dizionario italiano dell'uso, a cura di Tullio De Mauro, Torino, UTET, 2000; Moliner = M. Moliner, Diccionario de uso del español, Madrid, Gredos, 3ª . edición, 2007; RAE = Diccionario de la Lengua Española, Madrid, RAE, 2001; Sabatini-Coletti = F. Sabatini e V. Vittorio Coletti, Dizionario della lingua italiana, Milano, Rizzoli-Larousse, 2005; Salamanca = Diccionario Salamanca de la lengua española, Madrid, Santillana, 1996; Seco = M. Seco, O. Andrés y G. Ramos, Diccionario del Español actual, Madrid, Aguilar, 1999; Zingarelli = N. Zingarelli, Vocabolario della lingua italiana, Bologna, Zanichelli, Bologna, 2013. Inoltre: Bosque-Demonte = I. Bosque Muñoz y V. Demonte Barreto, Gramática 
sinonimici, varietà regionali, neologismi ecc.) e spesso abbiamo dovuto prendere decisioni per risolvere i problemi relativi alla disparità del trattamento grammaticale con cui le due lingue, italiano e spagnolo, sono solite descrivere e classificare i principali elementi dell'enunciato. Giacché la redazione di un lemma è prima di tutto una descrizione grammaticale e quindi una classificazione di una struttura di per sé astratta, ci siamo chiesti in che modo avremmo potuto rendere questa descrizione il più funzionale possibile sia alla lingua del lemma di entrata sia alla lingua di arrivo del traducente corrispondente, fissando come prioritario il tentativo di adeguare tra di loro i sistemi tassonomici grammaticali delle due lingue, senza tuttavia perderne di vista le caratteristiche e l'uso che si è consolidato nell'insegnamento grammaticale. I nomi dei complementi e i verbi transitivi / intransitivi sono le questioni che ci hanno dato più filo da torcere sia perché si presentano con altissima frequenza sia perché rispecchiano un modus operandi assai diverso nelle due lingue. Per l'italiano la classificazione dei complementi è ritagliata sulla base della grammatica latina e questo fatto condiziona non poco la terminologia grammaticale che segue delle norme diverse rispetto allo spagnolo; e così pure nella descrizione dei verbi, $i$ transitivi e gli intransitivi non hanno un eguale trattamento nelle due lingue, sia per l'uso diverso in italiano rispetto allo spagnolo di verbi apparentemente coincidenti, sia perché in spagnolo spesso la funzione intransitiva del verbo è trattata con l'indicazione «Absoluto» o con la sigla «U. t. c. intr.» («Usado también como intransitivo»).

\section{LA CLASSIFICAZIONE DEI COMPLEMENTI}

2.1. Consideriamo il primo punto: la classificazione dei complementi. E prendiamo come criterio di classificazione il modo di collegamento. Distinguiamo cioè i complementi che sono collegati al termine da cui dipendono per mezzo di una preposizione (complementi indiretti o obliqui) da quelli che sono collegati al termine da cui dipendono senza preposizione (complementi diretti). E già qui si presenta il primo problema, dal momento che nella grammatica spagnola è chiamato «Complemento Indirecto» (CI) il Complemento di Termine (caso dativo) e «Complemento Directo» (CD) il Complemento Oggetto (caso accusativo), per cui questa apparente coincidenza della terminologia grammaticale tra le due lingue («complemento directo / indirecto»), rischia di complicare, anziché chiarire i concetti ai nostri studenti. In Spagna l'analisi grammaticale distingue inoltre:

descriptica de la lengua española, Madrid, Espasa, 1999; Carrera Díaz = M. Carrera Díaz, Grammatica spagnola, Bari, Laterza, 2010; Renzi - Salvi = Grande grammatica italiana di consultazione, a cura di Lorenzo Renzi e Giampaolo Salvi, Bologna, Il Mulino, 1981, 3 voll.; Serianni = L. Serianni, Italiano. Grammatica, sintassi, dubbi, con la collaborazione di A. Castelvecchi, Torino, Garzanti, 2000. 
«Sujeto», corrispondente al Soggetto (caso nominativo)

«Complemento de Régimen preposicional», che può corrispondere grosso modo al Complemento di Specificazione (caso genitivo)

«Atributo», corrispondente al Complemento predicativo del soggetto «Complemento Predicativo», corrispondente al Complemento predicativo dell'oggetto

«Complemento Circustancial» che funziona più o meno come una categoria generale non definita numericamente, all'interno della quale gli studenti spagnoli sono abituati a riconoscere il «Complemento de lugar» (Complemento di luogo), il «Complemento de tiempo» (Complemento di tempo), il «Complemento de modo» (Complemento di modo) e il «Complemento de instrumento» (Complemento di mezzo).

Notiamo come le differenti terminologie siano il riflesso anche di un differente approccio all'analisi grammaticale, almeno a livello di istruzione scolastica. Là dove la scuola italiana tende a distinguere nettamente e con diverse denominazioni i vari tipi di complemento, in questo fortemente legata alla tassonomia grammaticale del Latino, (Complemento di tempo determinato/ indeterminato, di tempo continuato, di stato in luogo, di moto da /a/ per luogo, di specificazione, di modo, di compagnia, di unione, ecc.), quella spagnola ricorre all'etichetta più generica di «Complemento Circustancial».

Nella pratica lessicografica spesso ci siamo chiesti quale sistema adottare per descrivere in modo chiaro e omogeneo le funzioni dei complementi e quale fosse la terminologia migliore da usare, tenendo presenti le esigenze del nostro utente tipo, nonché i riferimenti grammaticali a lui più familiari.

Il problema si è presentato in modo manifesto nella redazione dei lemmi grammaticali, in particolar modo delle preposizioni.

Non riuscendo a trovare una soluzione convincente, comune ad entrambe le lingue, e partendo dalla convinzione che la descrizione della lingua deve essere funzionale a quella particolare lingua, abbiamo deciso di redigere i lemmi spagnoli in un modo e quelli italiani in un altro, fermo restando il criterio, vigente in tutto il dizionario, del raggruppamento delle diverse accezioni, o in questo caso delle funzioni, in base all'esito nella lingua d'arrivo: nella parte italianospagnolo abbiamo classificato le funzioni ricorrendo ai diversi complementi, mentre nella parte spagnolo-italiano abbiamo adottato una dizione di tipo semantico.

Per la preposizione italiana DA, per esempio, abbiamo raggruppato le differenti funzioni in base ai due traducenti (POR e DE) nei quali la preposizione italiana può essere risolta in spagnolo, e all'interno dei due diversi traducenti abbiamo distinto le varie funzioni con l'indicazione tra parentesi quadra del complemento corrispondente seguita da un consistente apparato di frasi d'esempio. Questo è il risultato: 
DA prep. semplice

1 [compl. di causa efficiente] por: la casa fu abbattuta da un terremoto la casa fue derribada por un terremoto; non farti prendere dalla paura no te dejes llevar por el miedo - [compl. d'agente] è stato scoperto dai nostri ricercatori ha sido descubierto por nuestros investigadores; le frecce scagliate dagli arcieri las flechas lanzadas por los arqueros • [compl. di mezzo] giudicare dal comportamento juzgar por el comportamiento; l'bo saputo dalla radio me he enterado por la radio • [compl. di moto per luogo] riuscì a passare dalla finestra logró pasar por la ventana; da questa parte, prego! ipor aquí, por favor! 2 [compl. di moto da luogo] de: sono appena arrivato dalla Germania acabo de llegar de Alemania; quando esci da scuola? ¿cuándo sales del colegio? • [compl. di provenienza] discende da una famiglia nobile desciende de una familia noble; ho riceruto una lettera da un amico he recibido una carta de un amigo; questo dipende da lui esto depende de él - [compl. di separazione] togliere un chiodo dal muro quitar un clavo de la pared - [compl. di distanza] il benzinaio è a tre chilometri dal paese la gasolinera está a tres kilómetros del pueblo; siamo ancora molto lontani dalla verità aún estamos muy lejos de la verdad • [compl. di causa] sta tremando dal freddo está temblando de frío; ha la voce roca dal tanto urlare tiene la voz ronca de tanto chillar • [compl. di scopo] cavallo da corsa caballo de carreras; occhiali da sole gafas de sol • [compl. di limitazione] cieco da un occhio ciego de un ojo • [compl. di stima] un esame da trenta e lode un examen de matrícula de honor $\cdot$ [compl. di prezzo] un quaderno da due euro un cuaderno de dos euros $\cdot$ [compl. di quantità o misura] un pacco da mezzo chilo un paquete de medio kilo; un servizio da dodici tazze un juego de doce tazas • [compl. di qualità] ieri ho visto la ragazza dai capelli biondi ayer vi a la chica del pelo rubio.

Per la preposizione spagnola $\mathrm{DE}$ il procedimento è lo stesso, ma in questa parte del dizionario le varie funzioni della preposizione $\mathrm{DE}$ sono descritte con un'indicazione tra parentesi quadra che fa riferimento all'uso della parola per la quale la preposizione funge da collegamento. Fanno eccezione il «compl. de tiempo»e il «compl. partitivo»:

DE prep. [se combina con el art. el formando del; la contracción no se realiza ante topónimos, titulos de obras literarias y nombres comerciales] 1 [indicando posesión o pertenencia] di: usa el ordenador de su hermano usa il computer di suo fratello; la casa de la actriz la casa dell'attrice; la subida de los precios de los alimentos l'aumento dei prezzi degli alimentari $\cdot$ [con un nombre en aposición] el mes de agosto il mese di agosto; la estación de Florencia la stazione di Firenze • [indicando materia] he comprado una mesa de madera ho acquistato un tavolo di legno; un collar de perlas una collana di perle $\cdot$ [indicando contenido] un vaso de vino un bicchiere di vino - [indicando asunto o tema de que trata] me gusta mucho ver películas de aventuras mi piace molto vedere film di avventura $\cdot$ [compl. partitivo] comió un poco de sopa mangiò un po' di minestra $\cdot$ [compl. de tiempo] son amigos de la infancia sono amici d'infanzia; el arte de este siglo l'arte di questo secolo; a las ocho de la tarde alle otto di sera; trabaja de noche lavora di notte [...] [indicando un día concreto] di + giorno della settimana: me falta el periódico del domingo 
mi manca il giornale di domenica; en el periódico del próximo martes habrá un artículo interesante nel giornale di martedì ci sarà un articolo interessante [...] 2 [con v. que indican que uno no puede permanecer] da: lo han expulsado de la organización è stato espulso dall organizzazione; le han echado de clase l'hanno mandato fuori dall'aula • [indicando movimiento desde un lugar] llegar de Moscú arrivare da Mosca; volviamos del cole a las cinco de la tarde tornavamo da scuola alle cinque del pomeriggio - [indicando origen, proveniencia] descender de una familia hebrea discendere da una famiglia ebrea; no me esperaba esto de ti non mi aspettavo questo da te; recibir una carta de una amiga ricevere una lettera da un'amica; aquel coche viene nada menos que de Japón quell'auto viene niente meno che dal Giappone - [indicando separación] quitar un clavo de la pared togliere un chiodo dal muro; se ha divorciado del segundo marido ha divorziato dal secondo marito • [indicando distancia] está a diez kilómetros de la ciudad è a dieci chilometri dalla città; estar lejos de casa essere lontano da casa $\cdot$ [indicando distancia figurada] nada más lejos de la verdad que lo que ha publicado la prensa boy niente è più lontano dalla verità di quello che la stampa ha pubblicato oggi - [introduce un predicativo] de pequeño vivía en una casa de campo da piccolo abitava in una casa di campagna; de mayor seré actriz da grande farò l'attrice; de estudiante, me ocurrió un hecho increible da studente mi accadde un fatto incredibile - [indicando pertinencia] es de estúpidos comportarse de este modo è da stupidi comportarsi così; ¡esto es de locos! (è) roba da matti!; manos de artista mani da artista; tiene actitudes de maleducado ha atteggiamenti da maleducato - [indicando dependencia] la última decisión depende de él la decisione ultima dipende da lui; todo depende de la economía americana tutto dipende dall'economia americana • [indicando uso, finalidad] caballo de carreras cavallo da corsa; red de pesca rete da pesca; gafas de sol occhiali da sole; sala de baile sala da ballo; tarjeta de visita biglietto da visita; cepillo de dientes spazzolino da denti; máquina de coser macchina da cucire • [indicando precio o cantidad] un cuaderno de tres euros un quaderno da tre euro; un billete de diez un biglietto da dieci; un paquete de medio kilo un pacco da mezzo chilo; un paquete de seis una confezione $\mathrm{da}$ sei; un servicio de doce tazas un servizio da dodici tazze 3 [indicando una cualidad cuando se sobrentiende ser] di: un jersey de color verde un maglione di colore verde; son palabras de mal gusto sono parole di cattivo gusto; un reloj de mucha calidad un orologio di grande qualità; [cuando se sobrentiende tener] da, con: ayer vi a la chica de pelo rubio ieri ho visto la ragazza dai capelli biondi; un restaurante de nombre francés un ristorante dal nome francese; [con comparación biperbólica implícita] da: vida de pachá vita da pascià; llevar una vida de perros menare una vita da cani; cuello de jirafa collo da giraffa; adelanto de infarto sorpasso da infarto; cifra de vértigo cifra da capogiro 4 [indicando instrumento o medio] con: un viejo de bastón un vecchio col bastone; lo hizo caer de una patada lo fece cadere con un calcio 5 [en construcciones comparativas] di: pesa más de cinco kilos pesa più di cinque chili; (de entre) tra, di: ¡qué gane el mejor de vosotros! che vinca il migliore tra voi! 6 [indicando limitación] da, di: sordo de una oreja sordo da un orecchio; ciego de un ojo cieco da un occhio 7 [cuando va después de un adj. y antes de un pron.] non si traduce: ipobre de mí! povero me! • [delante de algunos apellidos] los señores de Rupérez i coniugi Rupérez • (en calidad de) está de 
profesor en la universidad è professore allıuniversità; las dos hermanas estaban de enfermeras en una clínica le due sorelle facevano le infermiere in una clinica $\mathbf{8}$ [cuando se sobrentiende vestido de] in: la chica de verde la ragazza in verde.

2.2. Nella comune pratica di insegnamento dell'italiano come LS a studenti ispanofoni si dovrebbe, a nostro parere, evitare di abbracciare l'uno o l'altro sistema di analisi grammaticale, e piuttosto far ricorso a entrambi e, quando è possibile, anche al latino, giacché il riferimento ai casi ci può aiutare a chiarire ai nostri studenti perché nella frase italiana «alla ragazzina ho regalato un libro» «alla ragazzina» è un complemento di termine (caso dativo), mentre nella frase spagnola «Llevé a la niña a la escuela» «a la niña» è un complemento oggetto (caso accusativo), malgrado in entrambi i casi il nome (ragazzina/niña) sia introdotto dalla preposizione A. Per far capire agli studenti ispanofoni la differenza tra complemento di termine e complemento oggetto, può sempre essere utile ricorrere alla trasformazione della frase nella forma passiva. Il ricorso ai casi latini, quando è possibile, può anche aiutare a far capire la differenza tra la preposizione DI/DA. Per cui nella frase italiana «Il padrone di casa mi ha aumentato l'affitto» «di casa» è un complemento di specificazione (caso genitivo), mentre nella frase «Sono uscito di (o da) casa alle 8»«di o da casa» è un complemento di moto da luogo (e / ex + ablativo).

\section{IL PROBLEMA RELATIVO AI VERBI TRANSITIVI E INTRANSITIVI.}

3.1. Per affrontare in modo più sistematico l'analisi dei verbi transitivi e intransitivi nelle due lingue, proviamo a riunire in alcune categorie $i$ verbi spagnoli che si differenziano, rispetto ai corrispondenti verbi italiani, riguardo a questo aspetto. Per lo spagnolo sono stati consultati RAE, Seco, María Moliner e Salamanca; per l'italiano GRADIT e Sabatini- Coletti.

3.1.1. Verbi semanticamente e foneticamente molto simili nelle due lingue che in italiano sono esclusivamente intransitivi, mentre dalla RAE sono classificati anche come transitivi. È il caso di DESAPARECER che RAE risolve così:

\section{desaparecer}

1. tr. Ocultar, quitar de la vista con presteza. U. t. c. intr. y c. prnl.

2. intr. Dejar de existir.

Da Seco questo verbo è classificato solo come intransitivo, mentre María Moliner dopo la prima accezione intransitiva ne registra una seconda, transitiva, con l'etichetta «ant» e la specificazione «acep. causativa» di «hacer desaparecer alguna cosa». In casi come questo, l'accezione transitiva del verbo spagnolo non viene proprio registrata per non creare dubbi e perplessità nei nostri studenti. Così in GDS: 


\section{desaparecer}

v. intr. 1 (de la vista) scomparire, svanire, dileguarsi: el carterista desapareció por los pasillos del metro il borseggiatore scomparve nei corridoi della metropolitana 2 (morir) scomparire, mancare: muchos de los amigos del abuelo ya han desaparecido molti degli amici del nonno sono scomparsi 3 (fg.) sparire, svanire, scomparire: últimamente muchos de mis bolígrafos han desaparecido como por arte de magia ultimamente molte delle mie penne sono sparite come per magia; con estos masajes me ha desaparecido el dolor de espalda con questi massaggi mi è scomparso il mal di schiena 4 (ser robado) sparire, scomparire: el ladrón hizo d. de su casa todas las joyas il ladro fece sparire dalla sua casa tutti i gioielli.

Allo stesso tipo di verbi appartiene anche CENAR che coincide con l'italiano CENARE.

Secondo l'uso della lingua spagnola, da RAE, Seco, María Moliner è registrato sia come intransitivo che come transitivo. In italiano CENARE è però solo intransitivo, mentre l'uso transitivo è da tutti segnalato come letterario e arcaico. Nel GDS i traducenti italiani hanno osservato questa particolarità del verbo spagnolo e per tanto nell'uso transitivo del verbo sono proposte delle locuzioni verbali con valore transitivo.

\section{cenar}

A. v.intr. cenare: los andaluces suelen c. alrededor de las diez de la noche gli andalusi sono soliti cenare intorno alle dieci di sera $\mathbf{B}$ v.tr. mangiare a (o per) cena, cenare con: ayer cenaron pollo asado y ensalada ieri a cena hanno mangiato pollo arrosto e insalata.

3.1.2. Verbi semanticamente e foneticamente molto simili nelle due lingue che in spagnolo sono solo intransitivi, mentre in italiano sono sia transitivi che intransitivi, con esiti molto diversi nei traducenti. È il caso del verbo CESAR che RAE e María Moliner registrano solo come intransitivo, mentre il Seco registra anche nell'accezione transitiva con l'etichetta «col» (colloquiale) con il significato di DESTITUIR (todo el mundo puede ser cesado de hoy por la mañana).

Il verbo CESAR è così trattato nel GDS:

\section{cesar}

v.intr. cessare, smettere: los efectos de la tormenta no cesarán en seguida gli effetti della tempesta non cesseranno subito; poco después cesó de bailar poco dopo ha smesso di ballare - cesar en un cargo (o empleo) lasciare un incarico (o un impiego): su estado de salud le obligó a c. en su cargo il suo stato di salute l'ha obbligato a lasciare il suo incarico • sin cesar senza sosta B v. tr. (fam.) (destituir) silurare, destituire: el presidente del club cesó al entrenador il presidente del club ha silurato l'allenatore. 
Il verbo CESSARE è così trattato nel GDS:

cessàre v. intr.1 [aus. essere] parar, cesar: la pioggia è cessata la lluvia ha parado 2 [aus. avere] (interrompere le attività) cerrar: l'azienda ha cessato la empresa ha cerrado B v. tr. 1 (porre fine) suspender, interrumpir: c. le ostilità suspender las hostilidades $\mathbf{2}$ (smettere) dejar - cessare di + inf. $\rightarrow$ dejar de + inf.: ha cessato di lavorare ha dejado de trabajar; poco dopo ha cessato di nevicare poco después paró de nevar - cessare il fuoco (Mil.) cesar el fuego • cessare l'attività cesar la actividad, cerrar • cessate il fuoco! (Mil.) jalto el fuego!

3.1.3. Verbi che semanticamente e foneticamente sono molto simili nelle due lingue e che in spagnolo sono registrati da RAE solo come transitivi mentre in italiano possono essere sia transitivi che intransitivi. È il caso di TENDER che RAE classifica solo come transitivo, anche se Seco e María Moliner ne registrano pure l'accezione transitiva. Così il RAE:

\section{tender}

1. tr. Desdoblar, extender o desplegar lo que está cogido, doblado, arrugado o amontonado. 2. tr. Echar a alguien o algo por el suelo de un golpe. 3. tr. Echar por el suelo algo, esparciéndolo. 4. tr. Extender al aire, al sol o al fuego la ropa mojada, para que se seque. 5. tr. Suspender, colocar o construir algo apoyándolo en dos o más puntos. Tender una cuerda. Tender la vía. Tender un puente. 6. tr. Alargar algo aproximándolo hacia alguien o hacia otra cosa. 7. tr. Propender, referirse a algún fin. 8. tr. Dicho de una persona o de una cosa: Tener una cualidad o característica no bien definida, pero sí aproximada a otra de la misma naturaleza. 9. tr. Preparar una trampa o un engaño contra alguien. Le tendió una emboscada. 10. tr. Arq. Poner el tendido en paredes y techos. 11. tr. Mat. Dicho de una variable o de una función: Aproximarse progresivamente a un valor determinado, sin llegar nunca a alcanzarlo. 12. prnl. Echarse, tumbarse a la larga. 13. prnl. Dicho de las mieses o de otras plantas: Echarse o abatirse.

\section{Così il GDS:}

tender v. tr. 1 (la ropa) stendere: tiende la colada en la terraza stende il bucato sulla terrazza 2 (una trampa, una emboscada) tendere, porre: cayó en la trampa que le habian tendido cadde nel tranello che gli avevano teso $\mathbf{3}$ (las manos, los brazos) tendere, allungare: me tendió la mano para saludarme mi tese la mano per salutarmi 4 (acercar) porgere, allungare: amablemente, me tendió el paraguas gentilmente, mi porse l'ombrello 5 (tumbar) stendere, sdraiare, distendere: lo tendieron en una camilla lo sdraiarono su una barella $\mathbf{6}$ (también Electr.) (extender en una superficie) stendere, tendere, tirare: $t$. un hilo stendere un filo 7 (también Electr.) (extender suspendido) tendere, tirare: t. un cable tendere un cavo 8 (tensar) tendere, tirare: los marineros tienden las cuerdas $\mathrm{i}$ marinai tirano le funi 9 (arcos, cuerdas de instrumentos) tendere: t. las cuerdas del violin tendere le corde del violino $\mathbf{1 0}$ (abrir, desplegar) stendere, spiegare, 
distendere: los vendedores tienden una manta con la mercancía i venditori stendono una coperta su cui espongono la merce $\mathbf{1 1}$ (esparcir, desparramar) sparpagliare, spargere, spandere: tiende las lentejas para limpiarlas sparge le lenticchie per pulirle $\mathbf{1 2}$ (yeso, argamasa, cemento) gettare $\mathbf{1 3}$ (una vía férrea) costruire 14 (las redes) calare, tendere 15 ( Am.) (la cama) fare, rifare $16(\mathrm{Am}$. (la mesa) apparecchiare, preparare $\checkmark$ tender la (o una) mano a al. (fig.) tendere una mano a qlcu., offrire una mano a qlcu. $\diamond$ tender un puente (también fig.) gettare un ponte $\mathbf{B} \mathrm{v}$. intr. 1 (evolucionar) tendere: la economía tiende a la expansión l'economia tende all'espansione - (tener propensión) t. al exceso, al bien tendere all'eccesso, al bene $\cdot($ Mat.) $x$ tiende a y x tende a y $\mathbf{2}$ (tener como finalidad) essere volto, volta, essere finalizzato, finalizzata, essere diretto, diretta: sus acciones tienden a atizar la polémica le sue azioni sono finalizzate a creare polemica 3 (tirar, aproximarse) tendere, volgere: un naranja que tiende al rojo un arancione che tende al rosso $\nabla$ tender a mejorar tendere a migliorare: la situación tiende a mejorar la situazione tende a migliorare; ‘de tiempos volgere al bello, tendere a migliorare.

3.1.4. Verbi che semanticamente e foneticamente sono molto simili nelle due lingue e che nelle corrispondenti descrizioni grammaticali, possono essere classificati da alcuni come intransitivi da altri come «abs / ass.». È il caso di COCINAR e CUCINARE. Nel GDS è stata adottata la dicitura [absol.], secondo la classificazione di Seco e di Maria Moliner, mentre RAE ricorre alla dicitura «U. t. c. intr.»:

Cocinar v.tr. 1 cucinare: me encanta c. platos creativos mi piace moltissimo cucinare piatti creativi - [absol.] aprendió a c. muy joven imparò a cucinare quando era molto giovane $\mathbf{2}$ (fg. fam.) apparecchiare, preparare, cucinare: se está cocinando algo gordo si sta apparecchiando qualcosa di grosso.

3.1.5. Verbi che semanticamente sono molto distanti nelle due lingue e che sono registrati esclusivamente come transitivi, come il verbo COBRAR. Nelle accezioni in cui non è previsto il Complemento oggetto RAE ricorre all'indicazione «U. t. c. intr.», Seco e María Moliner ricorrono rispettivamente all'indicazione «Frec. Abs.» (Frecuentemente Absoluto) e «Abs.» (Absoluto). Nel nostro dizionario bilingue, per distinguere i diversi esiti nella lingua di arrivo, si è scelto di forzare la classificazione grammaticale spagnola e di inserire anche l'uso intransitivo del verbo, seguendo in questo modo la classificazione del Seco. Questo il risultato nel GDS:

cobrar A v.tr. 1 (recibir dinero) ricevere, incassare: si ganan la liga, los jugadores cobrarán una prima adicional se vincono lo scudetto i giocatori riceveranno un premio addizionale; he cobrado mil euros de adelanto ho incassato mille euro di anticipo 2 (pensión, salario) prendere, percepire: en esta empresa cobramos el sueldo a final de mes in questa ditta prendiamo lo stipendio alla fine del mese; mi madre cobra una pensión de viudedad mia madre percepisce una pensione 
di reversibilità • [absol.] j̇cuánto cobra un profesor de instituto en Italia? quanto prende un insegnante di scuola superiore in Italia? 3 (Econ.) riscuotere, incassare: hay que presentar una solicitud para c. los fondos comunitarios bisogna presentare una richiesta per riscuotere i contributi europei; era un cheque al portador muy difícil de c. en efectivo era un assegno al portatore molto difficile da incassare in contanti $\mathbf{4}$ (pedir dinero por algo) chiedere, prendere: por un masaje cobra treinta y seis euros per un massaggio chiede trentasei euro $\mathbf{5}$ (adquirir algo no material) acquistare, assumere: en estos uiltimos años la cultura del ocio ha cobrado auge in questi ultimi anni la cultura del tempo libero ha acquistato grande importanza; sus ojos cobraron un brillo especial i suoi occhi assunsero una luce particolare 6 (sentimientos) sentire, provare: empezó a c. cariño por aquella criatura incominciò a sentire affetto per quella creatura 7 (cuerda, soga) ritirare, recuperare 8 (en la caza: recoger las piezas) riportare $\mathbf{9}$ (cazar) prendere: cobraron dos ciervos hanno preso due cervi $\boldsymbol{B}$ v.intr. (fam.) $\mathbf{1}$ (recibir castigo corporal) prenderle, buscarle: ;si no te callas, cobrarás! se non stai zitto, le prendi! 2 (pedir precio) avere prezzi alti: en esta tienda cada vez cobran más caro in questo negozio hanno prezzi sempre più alti 3 (tirar de una cuerda) recuperare (tr.), ritirare (tr.): c. de una cuerda recuperare una corda $\nabla$ cobrar algo a al. fare pagar qlco. a qlcu., farsi pagare: nosotros le cobramos solo el tiempo efectivo de cada llamada noi le facciamo pagare soltanto la durata effettiva di ogni chiamata; $e l$ ayuntamiento pretende c. los servicios prestados il comune pretende di farsi pagare per i servizi prestati $\nabla$ cobrar algo a al. por (o de) algo fare pagare a qlcu. qlco. per qlco.: el banco me cobró un interés desorbitado por el préstamo la banca mi ha fatto pagare degli interessi spropositati per il credito.

3.2. Come per la descrizione e la terminologia da adottare per i complementi, così anche per la descrizione grammaticale dei verbi transitivi e intransitivi non siamo approdati a una sistematizzazione della questione, o quanto meno a una prassi risolutiva dei problemi che possano sorgere nel corso della pratica docente. Né tanto meno pretediamo di aver dato qui una esemplificazione esaustiva delle categorie di verbi più significativi della grammatica contrastiva spagnolo-italiana.

Con questa proposta di analisi di verbi transitivi e intransitivi nelle due lingue vorremmo più modestamente mettere in comune le esperienze e le soluzioni alle quali siamo giunti durante il lungo processo del nostro lavoro lessicografico. In estrema sintesi potremmo dire che nella descrizione grammaticale dei verbi, accanto alla classificazione di verbo transitivo/intransitivo è necessario introdurre anche quella di transitivo con valore assoluto, quando cioè il verbo funziona perfettamente anche senza l'esplicitazione del complemento oggetto. L'aggettivo assoluto, applicato al verbo transitivo, oltre ad essere termine comune alle due lingue (ass./abs) è anche quello che meglio ne descrive questa caratteristica; mentre la definizione «usato anche come intransitivo» può indurre in errore i nostri studenti e comunque aumentare la difficoltà a riconoscere questa particolare caratteristica del verbo. 